\title{
SALUD
}

\section{Construcción de las bases curriculares regionales para la profesionalización de la gestión sanitaria. Proyecto LASALUS “Diseño y desarrollo de un simulador para mejorar la gestión en Hospitales y Centros de Salud"}

\author{
Mónica Ruoti Cosp¹, Eduardo Velázquez², Daniel Franco ${ }^{3}$, Raquel \\ Araujo $^{4}$, Santiago Spadafora ${ }^{5}$
}

\section{Resumen}

Introducción: Se presentan resultados de la línea de base del proyecto LASALUS, que busca construir bases curriculares regionales para la profesionalización de la gestión sanitaria. La profesionalización en gestión sanitaria es fundamental para el logro de las metas del sistema de salud y por la falta de profesionales preparados para ocupar cargos directivos en las instituciones hospitalarias del país, se hace necesario ofertar cursos de especialización de modalidad virtual, que facilitaría la capacitación de profesionales de la salud. Proyecto interdisciplinario que articula 4 universidades europeas y 6 latinoamericanas más un consorcio de Bioingeniería e Informática.

Objetivo: Describir la experiencia del proyecto LASALUS cofinanciado por la Comunidad Europea en el marco del Programa Erasmus+.

Material y Método: Es una investigación acción participativa. Ha requerido de la participación activa de los miembros del proyecto para la construcción colectiva de las bases curriculares por medio de un trabajo en equipo multidisciplinario. En su fase analítica, se aplicaron técnicas cualitativas (revisión documental, entrevistas semi-estructuradas y grupos focales) y cuantitativas (encuesta web), y posteriormente se realizaron 5 talleres de trabajo.

Resultados: En una primera etapa 1ra. etapa se realizó una investigación como línea de base identificando opiniones y experiencias de los distintos grupos de actores claves en relación a la formación en gestión sanitaria. En la segunda etapa (2016), una pasantía en las Universidades Europeas de Navarra

\footnotetext{
1. Universidad Iberoamericana (UNIBE). Dirección de Investigación.

2. Universidad Iberoamericana (UNIBE). Facultad de Postgrados.

3. Universidad Iberoamericana (UNIBE).

4. Universidad Iberoamericana (UNIBE). Facultad de Ciencias de la Salud.

5. Universidad ISALUD. Buenos Aires. Argentina.

"El presente trabajo ha sido elaborado con la asistencia de la Unión Europea. El contenido de la misma es responsabilidad exclusiva de los autores responsables y en ningún caso debe considerarse que refleja los puntos de vista de la Unión Europea".
}

E-mail:monicaruoti@gmail.com

DOI: 10.26885/rcei.foro.2018.138 


\section{Bases curriculares regionales para la profesionalización de la gestión sanitaria. Ruoti et al.}

(Pamplona, España), Escuela de Altos Estudios en Salud Pública (Rennes, Francia) y de Pavia (Italia) para la transferencia de conocimientos, el diseño y desarrollo de un simulador. En el 2017, un Taller para la construcción de las bases curriculares regionales en San Salvador - El Salvador donde se elaboró el perfil del estudiante, se diseñó un plan de estudios iidentificando elementos pedagógicos y didácticos y la construcción de un contexto curricular y componentes del escenario donde se va a desarrollar la propuesta educativa que está compuesto por 9 Módulos: Bases de la Gestión de Servicios de Salud en base a resultado, Planificación de los Servicios de Salud, estrategia operativa y procesos, Manager y gestión del talento humano, Gestión Financiera y evaluación por resultados, Gestión de la calidad y riesgos asociados con la atención de la salud, Toma de decisiones basada en la evidencia, Gestión por proceso, Infraestructura, Tecnología y Comunicación y Trabajo Final de Grado. Tendrá una carga horaria regional: 270 horas (30 horas por módulos) y finalmente un Curso taller para Tutores y Contenidistas para e-learning realizado en la UNIBE para la formación de formadores. Actualmente se está realizando el curso en forma preliminar liderado por la Universidad Isalud (Argentina) para futuros tutores del mismo y se espera ofrecer el curso en el año 2019.

Conclusiones: La profesionalización en gestión sanitaria es fundamental para lograr las metas del sistema de salud. El curso contribuirá a mejorar la calidad educación superior de los profesionales que trabajan en las instituciones de la salud a través del desarrollo e implementación de un nuevo plan de estudios en la gestión de la asistencia sanitaria y a mejorar el nivel de competencias y habilidades para el diseño e implementación de planes de estudios de postgrado con componentes de e-learning y la formación basada en la simulación.

Palabras clave: bases curriculares, gestión sanitaria, profesionalización.

\section{Referencias}

Agüera, E., Alfageme, M. B. \& Calderón, M. D. (2005). Educación superior e innovaciones. Revista Iberoamericana de Educación, (36). Recuperado de https://es.scribd.com/document/186068665/1015Ordaxrieoei.org/ deloslectores/10150rdax.pdf

Bustos Sánchez, A., Coll, S. C. (2010). Los Entornos Virtuales Como Espacios De Enseñanza Y Aprendizaje Una perspectiva psicoeducativa para su caracterización y análisis. Revista Mexicana de Investigación Educativa, 163-184. Recuperado de http:// www.scielo.org.mx/pdf/rmie/v15n44/v15n44a9.pdf

Cataldi, Z., Lage, F., Dominighin, C. (2013). Fundamentos para el uso de simulaciones en la enseñanza. Revista de Informática Educativa y Medios Audiovisuales, 10(17), p. 8-16. Recuperado de http://laboratorios. fi.uba.ar/lie/Revista/Articulos/101017/A2mar2013.pdf

LASALUS Proyect (2017). Boletín de noticias LASALUS. Recuperado de http:// www.lasalus.org.ar/lasalusproject/newsletter022016.htmls 